Article type : Original Manuscript

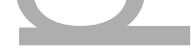

\title{
Systemic comorbidities are associated with medication-related osteonecrosis of the jaws: case-control study
}

\section{*Kelly McGowan}

School of Dentistry and Oral Health

Griffith University

Southport, Queensland, 4222, Australia

and School of Dentistry

University of Queensland

Herston, Queensland, 4029, Australia

Email: k.mcgowan@uq.edu.au

\section{Caroline Acton}

Oral \& Maxillofacial Surgery Department

Royal Brisbane and Women's Hospital

Herston, Queensland, 4029, Australia

\section{Saso Ivanovski}

School of Dentistry

University of Queensland

Herston, Queensland, 4029, Australia

This is the author manuscript accepted for publication and has undergone full peer review but has not been through the copyediting, typesetting, pagination and proofreading process, which may lead to differences between this version and the Version of Record. Please cite this article as doi: $10.1111 / 0 D I .13046$

This article is protected by copyright. All rights reserved 


\section{Newell W. Johnson}

Menzies Health Institute Queensland and

School of Dentistry \& Oral Health

Griffith University

Southport, Queensland, 4222, Australia

and Dental Institute, King's College London

\section{Robert S. Ware}

Menzies Health Institute Queensland

Griffith University

Southport, Queensland, 4222, Australia

\section{* Corresponding author}

\section{Running title: Systemic comorbidities predict MRONJ}

\section{Date of Submission: $28^{\text {th }}$ August, 2018}

\section{Number of figures and tables: 4}

Table 1: Distribution of demographics and study characteristics between cases and controls

Table 2: Association between comorbid conditions and MRONJ disease status

Table 3: Association between CPS and risk of MRONJ

Figure 1: Distribution of cases and controls in each CPS category (Attached separately)

Key Words: risk assessment, comorbidity, polypharmacy, bone density conservation agents, cancer, osteoporosis.

\footnotetext{
ABSTRACT

Objectives: Medication-related osteonecrosis of the jaws (MRONJ) is a serious condition developed in up to $15 \%$ of patients who take antiresorptive medications. Its underlying pathogenesis remains unclear. The association between systemic comorbidities and MRONJ was investigated.

Subjects and Methods: A case-control study was conducted in Brisbane, Australia. Hospital records were used to identify 68 cases of MRONJ between January 2003- March 2017. Each case was individually matched to 3 controls (204 in total) according to sex, age, primary
} 
disease, and type and duration of antiresorptive therapy. Data on patient demographic, social, and clinical characteristics were collected. Systemic comorbidities and medications were quantified as a Comorbidity Polypharmacy Score (CPS). Associations were investigated using conditional logistic regression.

Results: The CPS calculated for patients who developed MRONJ (mean \pm SD $=20.2 \pm 5.1$ ) was significantly higher than for controls (12.9 \pm 4.6$)$. Multivariable analysis determined a significant relationship between CPS and the presence of $\mathrm{MRONJ}(\mathrm{OR}=1.5 ; 95 \% \mathrm{CI}=1.3,1.8$, $\mathrm{p}<0.001)$.

Conclusions: Patients with multiple systemic comorbidities and high levels of polypharmacy were more likely to develop MRONJ. The CPS is a simple and effective tool to quantify the risk of MRONJ attributed to a patient's systemic condition, and should be considered in conjunction with the patient's oral health to assess the overall risk of MRONJ.

\section{INTRODUCTION}

Medication-related osteonecrosis of the jaws (MRONJ) has remained a largely enigmatic condition despite considerable research into its underlying pathogenesis since the first case series was published in 2003 (Marx, 2003). The prevalence of MRONJ is estimated to be between $0.001 \%-0.01 \%$ for osteoporotic patients, and $1 \%-15 \%$ for oncology patients (Khan et al., 2016). Medications associated with reported cases of MRONJ include bisphosphonates, denosumab, sunitinib, and bevacizumab, and possibly azacitidine, imatinib, everlimus, ipilimumab, and ziv-aflibercept (Owosho et al., 2018). It remains unclear why a small minority of patients will develop areas of jawbone necrosis during antiresorptive (AR) or antiangiogenic (AA) therapy, with therapy dose, duration and potency likely to influence the risk of MRONJ (Ruggiero et al., 2014). Importantly, however, high or sustained doses of implicated medications are not necessary for this disease to develop (Aljohani et al., 2017). There are a number of theories regarding the pathogenesis of MRONJ, with varying levels of evidence to support the following as contributing factors: osteocyte apoptosis (Roelofs et al., 2010); osteoclast suppression (Allen \& Burr, 2008); inhibition of blood vessel formation (Santini et al., 2003); obstruction of the vasculature (Favia et al., 2009); suppression of soft tissue healing (Reid et al., 2007), impaired immunity (Pazianas, 2011), genetic risk factors; (Choi et al., 2015), and infection or inflammation as a result of oral biofilms (Sedghizadeh et al., 2008). The role of oral health, and in particular dental extractions, has been extensively reported. While removal of a tooth in a patient on AR therapy is the most commonly reported initiator of MRONJ (McGowan et al., 2018), most patients on ARs will heal normally after a 
dental extraction (Mozzati et al., 2012). Periodontal disease, denture trauma, dental implants, and tori have also been reported as possible dental risk factors for MRONJ (McGowan et al., 2018). The pattern of disease suggests that patient susceptibility is key, with two constituent parts to a patient's risk profile: 1) their overall dental health, and 2) their overall systemic health. This study is specifically interested in systemic risk indicators, some of which may be true risk factors, for MRONJ.

A number of potential systemic risk factors for MRONJ have been previously reported, including chemotherapy, diabetes, glucocorticoid therapy, anemia, renal disease and smoking (Khan et al., 2017; McGowan et al., 2018), however there is currently no evidence-based method to assimilate these potential risk factors into a clinically relevant format. In medicine, a number of comorbidity indices are used to predict which patients are likely to experience poor outcomes. The Comorbidity Polypharmacy Score (CPS) was developed in a population of trauma patients in 2011 (Evans et al., 2012; Evans et al., 2011), and has been validated in a number of other settings (Housley et al., 2015; Justiniano et al., 2015; Mubang et al., 2015; Nossaman et al., 2017; Tolentino et al., 2016; Tolentino et al., 2017). The CPS is the sum of the number of pre-MRONJ medications plus all known comorbidities, with the total score indicating the patient's cumulative severity. The incorporation of both the patient's medical and pharmacological history makes it a good candidate for use in MRONJ populations. The ease of administration makes it a feasible approach for the routine clinical dental setting.

The aim of this study was to investigate whether CPS was associated with the risk of MRONJ in patients taking AR medications. It was hypothesized that systemically unwell patients were at a higher risk of MRONJ, and therefore a higher CPS will be associated with an increased risk of MRONJ.

\section{METHOD}

Study Design

A case-control study was conducted in Brisbane, Australia, to compare the presence or absence of putative medical risk factors, as risk indicators, between cases with a clinical diagnosis of MRONJ and controls. Patients who were treated between 1 January 2003 and 30 March 2017 were eligible for inclusion. Patient records were reviewed at the Royal Brisbane \& Women's Hospital (RBWH), and at the Gold Coast University Hospital. Ethical approval for the study was obtained from the RBWH Human Research Ethics Committee (ID: EC00172, HREC/17/QRBW/242) and Griffith University Human Research Ethics 
Committee (Ref 2016/927). All patient information was de-identified to protect anonymity. The reporting of this study conforms to the STROBE statement (Von Elm et al., 2007).

\section{Case Selection}

Potential cases were identified from hospital records using International Classification of Disease and Related Health Problems (ICD-10-AM $9^{\text {th }}$ Edition) code K10.2, which represents inflammatory conditions of the jaw. As K10.2 includes a number of diseases (osteitis, osteomyelitis, osteonecrosis, osteoradionecrosis, periostitis, and sequestrum of jaw bone), each chart was manually reviewed to confirm the clinical diagnosis. The following criteria were used to determine case eligibility:

1. Taking antiresorptive or antiangiogenic medications

2. Exposed bone in the oral cavity that did not heal within 8 weeks after identification by a health care provider

3. No history of radiation therapy to the craniofacial region

4. No history of malignant or metastatic disease of the jaws

5. Aged 18 years or older

6. Not pregnant or breastfeeding

\section{Control Selection}

Three controls were individually matched to each case on age, sex, primary disease, and AR therapy and duration. Controls were identified from hospital records using ICD-10-AM codes for breast cancer, prostate cancer, multiple myeloma, osteoporosis, Paget's disease, and rheumatoid arthritis. Age and sex were matched to eliminate possible confounding due to age-related decline in immune function, or any hormonal variation in risk. The type of AR drug prescribed and its dosage and duration were matched as closely as possible, as these variables are known to increase the risk of MRONJ (Ruggiero et al., 2014). Where it was not possible to match the AR regimen exactly, the selected controls were required to have more potent or longer duration AR therapy in order to ensure that pharmaceutical factors were not reducing the risk of MRONJ in controls relative to their cases. Sex and age (to within 5 years) were matched to eliminate hormonal variations or age-related factors as potential confounders. Two potential controls who had received teriparatide (Forteo) for osteoporotic fractures were replaced, as teriparatide has been used in the treatment of MRONJ (Cheung \& Seeman, 2010) and may have offered some protection against MRONJ in these patients. 


\section{Variables \& Data Collection}

The primary exposures of interest were co-existing chronic diseases, and pharmacological therapies. For cases, all data were collected from the most recent time point available prior to their MRONJ diagnosis, while the most current data were recorded for controls. The primary data source was clinical notes, but all available sources were used to limit potential information bias due to incomplete notes when possible. Comorbidities were determined from computer-generated general practitioner referrals or specialist outpatient clinic letters. Medications were recorded from the patient's Medical Action Plan (MAP) provided by the hospital pharmacist or an outpatient medication list. Data on domestic living arrangements, tobacco and alcohol use were collected using the Patient Risk Assessment form or preanesthetic record. Patients were defined as cohabiting if they were married or in a de facto relationship. Alcohol abuse was determined from the hospital risk assessment form as either daily alcohol use or $>6$ drinks/session. Tobacco use was recorded as one of four categories: never, quit within the past five years, quit more than five years previously, or current user. Each patient's postcode and marital status was collected from their admission data. Postcodes were used to calculate the Index of Relative Socio-Economic Disadvantage and Advantage for each subject, using the Socio-Economic Indexes for Areas Index (ABS, 2018).

AR therapy was recorded from the MAP, outpatient medication lists, chemotherapy orders, or infusion records. Systolic and diastolic blood pressure, pulse rate, and anthropometric variables were collected from general observation charts, chemotherapy orders, or preanesthetic assessments. Body Mass Index was calculated using standard formula and categorized as: $<18.5=$ below healthy weight range, $18.5-25=$ healthy weight range, and $>25$ $=$ above healthy weight range.

Each patient's comorbidities were converted into the "C" component of the CPS by adding all identified comorbidities together. Identified comorbidities are listed in Supplemental Appendix 1. Data on polypharmacy were collected as a list of medications, and converted into the "P" component of the CPS by adding all identified medications together. As supplementary medications were sometimes prescribed as individual supplements (calcium, magnesium, glucosamine, iron) and sometimes as combinations or multi-vitamins, patients scored one point if they were prescribed any supplementary medication. Regular pain medications were scored similarly, excluding morphine or oxycodone that were scored individually. Each comorbidity or medication was assigned one point, and the " $C$ " and "P" 
scores were added together to give an overall CPS. CPS scores were then grouped according to previously identified categories: mild (0-7 points), moderate (8-15 points), severe (15-21 points) or morbid (>21 points) (Evans et al., 2011; Evans et al., 2012).

\section{Statistical methods:}

Sample size calculations were based on the assumption of a binary exposure variable (categorised as high or low risk). To calculate the detectable difference in MRONJ cases by risk status we assumed $60 \mathrm{MRONJ}$ cases would be identified, with three matched controls per case. Prior data indicated the average probability of a control being classified as high risk was 0.5 , and the correlation coefficient for being high risk between matched cases and controls was 0.3 . With $80 \%$ power and alpha $=0.05$ we would be able to detect a significant difference in risk groups if the odds ratio observed was 2.2 or greater.

Statistics were summarised as frequencies (percentage). Demographic, social, and clinical characteristics of cases and controls were compared using the chi-square test. Conditional logistic regression was used to identify factors associated with MRONJ. Models were conditioned on their individually-matched case-control groups. Unadjusted odds ratios (OR) and $95 \%$ confidence intervals (CI) were calculated where the exposure was CPS score and the outcome was MRONJ. Adjusted odds ratios (aOR) were calculated using multivariable conditional logistic regression. Potentially confounding variables included in multivariable analysis were smoking and duration of AR therapy as these variables were identified during the univariable analysis. Disease groups that were significant in the univariable analysis but were captured in the CPS were not adjusted for in the multivariable models. A pre-specified stratified analysis of oncologic and non-oncologic subgroups was conducted. Statistical analyses were conducted using IBM SPSS Statistics, Version 25 (IBM Corporation 2017 C), Aarmonk, NY, USA).

\section{RESULTS}

Overall, 68 cases of MRONJ were identified. Three controls were individually matched to each case (204 controls), with a total sample size of 272 . The age of patients ranged from 43 to 94 years, with a mean \pm SD age of $69.4 \pm 12.2$ years, and $59 \%$ of participants were female. Demographic, social, and clinical characteristics of cases and controls are compared in Table 1 , and show that the matching process was effective at preventing any significant differences in sex, age, primary disease, or type of AR therapy. There was no significant difference 
between socioeconomic position or living arrangements for cases and controls. There was a significant difference in the duration of AR therapy with controls tending to have received longer courses of ARs than cases, reflecting the decision making process in the matching protocol. Consequently, duration of AR therapy was included as a co-variable in multivariable analyses.

The systemic health of cases and controls were compared and results are presented in Table 2. Patients with non-insulin dependent diabetes mellitus, respiratory disease, cardiovascular disease, chronic kidney disease, blood disorders, mental illness, or who were current smokers had significantly greater odds of developing MRONJ. The distribution of chemotherapy was very similar between cases and controls, likely reflecting the standardised treatment recommendations for the matched primary diseases. Only 1 case and 3 controls were prescribed AAs, and therefore it was not possible to perform any meaningful analysis on the relative risk of MRONJ with AA medications in this cohort.

Associations between the comorbidity and polypharmacy components of the CPS, as well as the combined CPS, and disease status are presented in Table 3. There was a highly statistically significant difference in both the individual components and the overall CPS. The mean CPS score for cases was $20.2 \pm 5.1$ compared to $12.9 \pm 4.6$ for controls. The model showed that the odds of developing MRONJ increased by 1.5 for every 1 unit increase in CPS (95\%CI=1.6, 2.y; $\mathrm{p}<0.001)$, after adjusting for the potentially confounding variables of tobacco use and duration of AR therapy. Oncology cases and controls reported a mean CPS of $19.1 \pm 4.5$ and $12.4 \pm 4.5$ respectively, which was similar to the mean CPS of $20.9 \pm 5.7$ and $13.3 \pm 4.7$ calculated for the non-oncology cases and controls. When CPSs were grouped into severity categories, the odds of a morbid patient developing MRONJ were 12.7 times greater $(\mathrm{p}=<0.001)$ than that of a severe patient. The odds of a patient with a moderate CPS score developing MRONJ was 10 times lower than patients classified as severe $(\mathrm{p}<0.001)$. The distribution of cases and controls between CPS stratifications is shown in Figure 1.

\section{DISCUSSION}

The CPS is a simple and effective tool to quantify how the cumulative severity of disease in an individual patient relates to their risk of developing MRONJ. While individual diseases are separately associated with the development of MRONJ, an obvious advantage of the CPS is the ease of clinical application and interpretation. 
The CPS offers a number of advantages over other comorbidity measures for MRONJ. Two other comorbidity indices have been used in previous MRONJ research and support the association between multiple comorbid conditions and increasing MRONJ risk. Lapi et al. used the Charlson Comorbidity Index (CCI) as a measure of chronic severity and found that MRONJ cases reported significantly higher CCIs compared to controls (Lapi et al., 2013). Kwon et al. used individual components of the CCI to adjust for confounding and reported that cases more frequently recorded CCI conditions but no formal analysis of this difference was conducted (Kwon et al., 2015). Based on these published reports, the use of the CCI was piloted in this project but was deemed unsuitable due to the complexity of the criteria (Charlson et al., 1994) and the time taken to accurately calculate a result. Baillargeon et al. used a modified version of the Elixhauser method (Elixhauser et al,. 1998) to quantify comorbidities and also found that MRONJ cases had significantly more comorbidities than controls (Baillargeon et al., 2011). The Elixhauser method uses International Classification of Disease (ICD) codes which dental practitioners do not routinely access, and for this reason it was also not suitable for this project.

Within the CPS, the inclusion of the patient's pharmacology profile in addition to their comorbid conditions better reflects previously reported risk factors for MRONJ (Khan et al., 2016; McGowan et al., 2017). The simplicity of the system makes it a feasible approach for routine use in dental practice. This study found that the association between CPS and MRONJ was similar between oncology and non-oncology patients and therefore it could be applied to all patients without the need for complicated weightings for different patient groups. It is a validated predictor of outcomes (Evans et al., 2012; Evans et al., 2011), readmissions (Housley et al., 2015), in-hospital complications (Justiniano et al., 2013), and mortality (Nossaman et al., 2017) in older trauma patients, as well as hospital mortality and readmissions for surgical patients (Tolentino et al., 2017). It was also shown to be a more accurate prognostic tool than the CCI or chronologic age for overall survival in elderly patients with cancer of the oropharynx (Caparrotti et al., 2016). The authors suggested the superiority of the CPS over the CCI may be due to the inclusion of polypharmacy, which better reflected the mortality risk than assessment of comorbidities alone (Caparrotti et al., 2016). 
The ability of the CPS to identifying high-risk patients has important clinical implications for the dental management of patients on AR therapies. It is well established that preventative dental measures significantly reduce the incidence of MRONJ (Bonacina et al., 2011; Mücke et al., 2016; Ripamonti et al., 2008; Vandone et al., 2012), and the CPS could be used to identify patients at the highest risk of MRONJ so that more frequent and intensive preventative care can be provided. Current guidelines state that dental implants can be considered for osteoporotic patients on AR therapy (Khan et al., 2015) but there is no validated method for determining which patients are suitable candidates. Similarly, a number of modifications to techniques for exodontia have been described to minimise the risk of MRONJ (Gaudin et al., 2015), but it is important for surgical planning to understand which patients are at increased risk of developing MRONJ. C-terminal telopeptide (CTX) scans have been previously investigated as a possible predictor of MRONJ risk (Marx et al., 2007) but have been subsequently shown to have no predictive value (Enciso et al., 2016). The clinical utility of the CPS should be further investigated as a rapid and non-invasive alternative to laboratory testing to assist clinicians in managing patients on AR therapies.

The careful matching of cases to controls on the basis of their primary disease and AR therapy was an important strength of this study. Eliminating the type and duration of AR therapy as confounding factors allowed this study to focus on the role of comorbidities and polypharmacy in determining MRONJ risk. Although the intention was to match for duration of AR therapy, it was not possible to achieve this in every instance. Where necessary, controls with longer durations of AR therapy were used. This unbalanced distribution of duration was reflected in the univariable analysis, where the odds ratios were biased downwards (i.e. towards the null). The use of retrospective hospital data was an important limitation and must be considered when interpreting the results. Errors within individual clinical records and missing data may have influenced the outcome. Although the sample size was calculated using a binary exposure variable, during data collection it was decided to treat CPS as an interval variable. Given that continuous exposure variables have higher power against fixed alternative hypotheses than binary exposure variables, we did not recalculate the sample size, and the highly significant results demonstrate that a sufficient number of participants were identified.

In this study, cases and controls received chemotherapy and corticosteroids in equal frequencies and the risk of MRONJ was not significantly associated with either medication 
type. This may reflect that medical treatment in Australia is regulated through clinical and therapeutic guidelines, and therefore matching patients by primary disease may have incidentally matched for certain systemic treatments. However, most of the data that supports a significant association between chemotherapy, corticosteroids and MRONJ comes from cohort studies (McGowan et al., 2018), and without a control group it is possible that this relationship has been overestimated. Our study suggests that chemotherapy and corticosteroids are common treatments in patients who receive antiresorptive therapy, and that the cumulative burden of disease and pharmacotherapy are more predictive of MRONJ risk than individual medications.

It is important to note that the CPS does not measure dental risk factors, which are a critical consideration when assessing the likelihood of an individual patient developing MRONJ. Australia has universal health coverage under Medicare but this does not extend to dental treatment. Almost half of the participants in this study did not have an available public dental record, and therefore the association between oral disease, dental treatment and MRONJ could not be determined within the present data set. Dental risk factors will be investigated in a future report utilizing the available dental data.

Only four participants had been prescribed antiangiogenics, and therefore future research should investigate other patient cohorts, such as colorectal cancer, renal cancer, and nonsmall cell lung cancer, to better understand the relationship between antiangiogenics and MRONJ. The use of hospital controls is often associated with the recruitment of a generally sicker population, and therefore the CPSs calculated in this study may be higher than those of patients taking AR therapies that are managed in general practice clinics. It would be important to investigate the utility of the CPS in MRONJ patients managed in private oral surgery clinics, as the cases identified in this study are likely to have more advanced stages of disease. The generalizability of these results to other patient populations is currently unknown, and it will be important to determine if the association between CPS results and MRONJ risks persists in other populations of patients taking AR therapies, such as patients managed outside of the hospital setting as well as populations from different countries and with different ethnicities. Future research should also investigate whether CPS is a predictor of MRONJ treatment outcomes. 
Results of this study suggest that a patient's risk of MRONJ increases significantly with systemic comorbidities and polypharmacy. The CPS offers a simple and effective method of quantifying this risk. Clinical assessment of the patient's dental risk factors should be used in conjunction with the CPS to estimate the overall risk of MRONJ.

A supplemental appendix to this article is available.

\section{ACKNOWLEDGEMENTS}

This study was supported by the Griffith University School of Dentistry and Oral Health.

Conflict of interest: K McGowan has received a speaker honorarium from Eli Lilly.

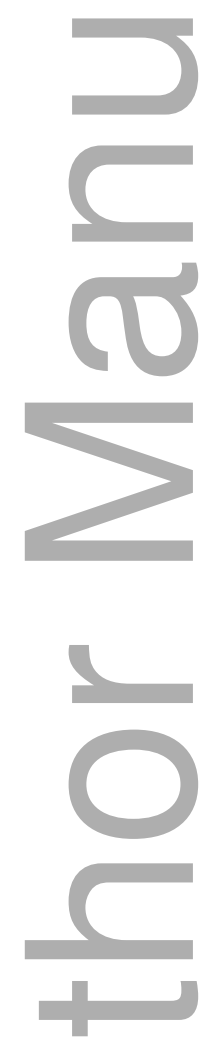

\section{REFERENCES}

Aljohani, S., Fliefel, R., Ihbe, J., Kühnisch, J., Ehrenfeld, M., \& Otto, S. (2017). What is the effect of anti-resorptive drugs (ARDS) on the development of medication-related osteonecrosis of the jaw (MRONJ) in osteoporosis patients: A systematic review. Journal of Craniomaxillofacial Surgery, 45(9), 1493-1502.

Allen, M.R., \& Burr, D.B. (2008). Mandible matrix necrosis in beagle dogs after 3 years of daily oral bisphosphonate treatment. Journal of Oral and Maxillofacial Surgery, 66(5), 987994. 
[ABS] Australian Bureau of Statistics. [Internet]. (2016). Census of population and housing: Socio-economic indexes for areas (SEIFA), Australia, 2016. Canberra: Australian Bureau of Statistics. [updated 2018 March 27; cited 2018 May 30]. Available from: http://www.abs.gov.au/ausstats/abs@.nsf/mf/2033.0.55.001

Baillargeon, J., Kuo, Y.F., Lin, Y.L., Wilkinson, G.S., \& Goodwin, J,S. (2011). Osteonecrosis of the jaw in older osteoporosis patients treated with intravenous bisphosphonates. Annals of Pharmacotheapyr, 45(10), 1199-1206.

Bonacina, R., Mariani, U., Villa, F., \& Villa, A. (2011). Preventive strategies and clinical implications for bisphosphonate-related osteonecrosis of the jaw: A review of 282 patients. Journal of the Canadian Dental Association, 77(5), b147.

Caparrotti, F., Huang, S., Ringash, J., Song, Y., Bayley, A., Bratman, S., .. Kim, J. (2016). Ep1093: Impact of comorbidity, polypharmacy and HPV status in elderly patient with oropharyngeal cancer. Radiother Oncol. 119:S526.

Charlson, M., Szatrowski, T.P., Peterson, J., \& Gold, J. (1994). Validation of a combined comorbidity index. Journal of Clinical Epidemiology, 47(11), 1245-1251.

Cheung, A., \& Seeman, E. (2010). Teriparatide therapy for alendronate-associated osteonecrosis of the jaw. New England Journal of Medicine, 363(25), 2473-2474.

Choi, H., Lee, J., Lee, J.H., Kim, J.H. (2015). Genetic association between VEGF polymorphisms and BRONJ in the Korean population. Oral Diseases, 21(7), 866-871.

Elixhauser, A., Steiner, C., Harris, D.R., \& Coffey, R.M. (1998). Comorbidity measures for use with administrative data. Medical Care. 36(1), 8-27.

Enciso, R., Keaton, J., Saleh, N., Ahmadieh, A., Clark, G.T., \& Sedghizadeh, P.P. (2016). Assessing the utility of serum c-telopeptide cross-link of type 1 collagen as a predictor of bisphosphonate-related osteonecrosis of the jaw: A systematic review and meta-analysis. Journal of the American Dental Association. 147(7), 551-560

Evans, D.C., Cook, C.H., Christy, J.M., Murphy, C.V., Gerlach, A.T., Eiferman, ... Beery, P.R. (2012). Comorbidity-polypharmacy scoring facilitates outcome prediction in older trauma patients. Journal of the American Geriatric Society, 60(8), 1465-1470.

Evans, D.C., Gerlach, A.T., Christy, J.M., Jarvis, A.M., Lindsey, D.E., Whitmill, M.L., ... Beery, P.R. (2011). Pre-injury polypharmacy as a predictor of outcomes in trauma patients. International Journal of Critical Illness and Injury Science. 1(2), 104.

Favia, G., Pilolli, G., \& Maiorano, E. (2009). Histologic and histomorphometric features of bisphosphonate-related osteonecrosis of the jaws: An analysis of 31 cases with confocal laser scanning microscopy. Bone, 45(3), 406-413. 
Gaudin, E., Seidel, L., Bacevic, M., Rompen, E., \& Lambert, F. (2015). Occurrence and risk indicators of medication-related osteonecrosis of the jaw after dental extraction: A systematic review and meta-analysis. Journal of Clinical Periodontology, 42(10), 922932.

Housley, B.C., Stawicki, S.P., Evans, D.C., \& Jones, C. (2015). Comorbidity-polypharmacy score predicts readmission in older trauma patients. Journal of Surgical Research, 199(1), 237 243.

Justiniano, C.F., Coffey, R.A., Evans, D.C., Jones, L.M., Jones, C.D., Bailey, J.K., ... Stawicki, S.P. (2015). Comorbidity-polypharmacy score predicts in-hospital complications and the need for discharge to extended care facility in older burn patients. Journal of Burn Care Research, 36(1), 193-196.

Justiniano, C.F., Eyans, D.C., Cook, C.H., Eiferman, D.S., Gerlach, A.T., Beery, P.R., ... Miller, S.F. (2013). Comorbidity-polypharmacy score: A novel adjunct in post-emergency department trauma triage. Journal of Surgical Research, 181(1), 16-19.

Khan, A.A., Morrison, A., Hanley, D.A., Felsenberg, D., McCauley, L.K., O'Ryan, F., ... Tetradis, S. (2015). Diagnosis and management of osteonecrosis of the jaw: A systematic review and international consensus. Journal of Bone and Mineral Research, 30(1), 3-23.

Khan, A.A., Morrison, A., Kendler, D.L., Rizzoli, R., Hanley, D.A., Felsenberg, D., ... Ruggiero, S.L. (2017). Case-based review of osteonecrosis of the jaw (ONJ) and application of the international recommendations for management from the International Task Force on ONJ. Journal of Clinical Densitometry, 20(1), 8-24.

Kwon, J.W., Park, E.J., Jung, S.Y., Sohn, H., Ryu, H., \& Suh, H. (2015). A large national cohort study of the association between bisphosphonates and osteonecrosis of the jaw in patients with osteoporosis: A nested case-control study. Journal of Dental Research, 94(9), s212s219.

Lapi, F., Cipriani, F., Caputi, A., Corrao, G., Vaccheri, A., Sturkenboom, M., ... Staniscia, T. (2013). Assessing the risk of osteonecrosis of the jaw due to bisphosphonate therapy in the secondary prevention of osteoporotic fractures. Osteoporosis International, 24(2), $697-705$.

Marx, R.E. (2003). Pamidronate (aredia) and zoledronate (zometa) induced avascular necrosis of the jaws: A growing epidemic. Journal of Oral Maxillofacial Surgery, 61(9), 1115-1117.

Marx, R.E., Cillo, J.E., \& Ulloa, J.J. (2007). Oral bisphosphonate-induced osteonecrosis: Risk factors, prediction of risk using serum ctx testing, prevention, and treatment. Journal of Oral Maxillofac Surgery, 65(12), 2397-2410. 
McGowan, K., McGowan, T., \& Ivanovski, S. (2018). Risk factors for medication-related osteonecrosis of the jaws: A systematic review. Oral Diseases, 24(4), 527-536

Mozzati, M., Arata, V., \& Gallesio, G. (2012). Tooth extraction in patients on zoledronic acid therapy. Oral Oncology, 48(9), 817-821.

Mubang, R.N., Stoltzfus, J.C., Cohen, M.S., Hoey, B.A., Stehly, C.D., Evans, D.C., ...Hoff, W.S. (2015). Comorbidity-polypharmacy score as predictor of outcomes in older trauma patients: A retrospective validation study. World Journal of Surgery, 39(8), 2068-2075.

Mücke, T., Deppe, H., Hein, J., Wolff, K.D., Mitchell, D.A., Kesting, M.R., ...Thalgott, M. (2016). Prevention of bisphosphonate-related osteonecrosis of the jaws in patients with prostate cancer treated with zoledronic acid - a prospective study over 6 years. Journal of Craniomaxillofacial Surgery, 44(10), 1689-1693.

Nossaman, V.E., Larsen, B.E., Digiacomo, J.C., Manuelyan, Z., Afram, R., Shukry, S., ... Angus, L.G. (2017). Mortality is predicted by comorbidity polypharmacy score but not Charlson Comorbidity Index in geriatric trauma patients. American Journal of Surgery. (in press) https://doi.org/10.1016/j.amjsurg.2017.09.011

Owosho, A.A., Liang, S.T.Y., Sax, A.Z., Wu, K., Yom, S.K., Huryn, J.M., \& Estilo, C.L. (2018). Medication-related osteonecrosis of the jaw (MRONJ): An update on the Memorial Sloan Kettering Cancer Center (MSKCC) experience and the role of pre-medication dental evaluation in the prevention of MRONJ. Oral Surgery Oral Medicine Oral Pathology Oral Radiology and Endodontology, 125(5), 440-445.

Pazianas, M. (2011). Osteonecrosis of the jaw and the role of macrophages. Journal of the National Cancer Institute, 103(3), 232-240.

Reid, I.R., Bolland, M.J., \& Grey, A.B. (2007). Is bisphosphonate-associated osteonecrosis of the jaw caused by soft tissue toxicity? Bone, 41(3), 318-320.

Ripamonti, C., Maniezzo, M., Campa, T., Fagnoni, E., Brunelli, C., Saibene, G., ... Cislaghi, E. (2008). Decreased occurrence of osteonecrosis of the jaw after implementation of dental preventive measures in solid tumour patients with bone metastases treated with bisphosphonates. The experience of the National Cancer Institute of Milan. Annals of Oncology, 20(1), 137-145

Roelofs, A.J., Coxon, F.P., Ebetino, F.H., Lundy, M.W., Henneman, Z.J., Nancollas, G.H., ... Kashemirov, B.A. (2010). Fluorescent risedronate analogues reveal bisphosphonate uptake by bone marrow monocytes and localization around osteocytes in vivo. Journal of Bone and Mineral Research, 25(3):606-616.

Ruggiero, S.L., Dodson, T.B., Fantasia, J., Goodday, R., Aghaloo, T., Mehrotra, B., \& O'Ryan, F. (2014). American association of oral and maxillofacial surgeons position paper on 
medication-related osteonecrosis of the jaw-2014 update. Journal of Oral and Maxillofacial Surgery, 72(10), 1938-1956.

Santini, D., Vincenzi, B., Dicuonzo, G., Avvisati, G., Massacesi, C., Battistoni, F., ... Altomare, V. (2003). Zoledronic acid induces significant and long-lasting modifications of circulating angiogenic factors in cancer patients. Clinical Cancer Research, 9(8), 28932897

Sedghizadeh, P.P., Kumar, S.K., Gorur, A., Schaudinn, C., Shuler, C.F., Costerton, J.W. (2008). Identification of microbial biofilms in osteonecrosis of the jaws secondary to bisphosphonate therapy. Journal of Oral and Maxillofacial Surgery, 66(4), 767-775.

Tolentino, J.C., Stoltzfus, J.C., Harris, R., Foltz, D., Deringer, P., Sakran, J.V., ... Moffatt-Bruce, S.D. (2017). Comorbidity-polypharmacy score predicts readmissions and in-hospital mortality: A six-hospital health network experience. Journal of Basic Clinical Pharmacy, $8(3): 98-103$.

Vandone, A., Donadio, M., Mozzati, M., Ardine, M., Polimeni, M., Beatrice, S., ... Scoletta, M. (2012). Impact of dental care in the prevention of bisphosphonate-associated osteonecrosis of the jaw: A single-center clinical experience. Annals of Oncology, 23(1), 193-200.

Von Elm, E., Altman, D.G., Egger, M., Pocock, S.J., Gøtzsche, P.C., Vandenbroucke, J.P., \& Strobe Initiative. (2007). The Strengthening the Reporting of Observational Studies in Epidemiology (STROBE) statement: guidelines for reporting observational studies. PLoS Medicine, 4(10), e296.

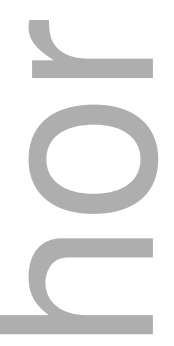

Table 1. Distribution of demographics and study characteristics between cases and controls

\begin{tabular}{|c|c|c|c|}
\hline \multirow[t]{2}{*}{ Characteristics } & Case $(\mathrm{N}=68)$ & Control $(\mathrm{N}=204)$ & $\mathbf{p}$ \\
\hline & $\mathrm{N}(\%)$ & $\mathrm{N}(\%)$ & \\
\hline Sex & & & 1.00 \\
\hline Male & $28(41.2)$ & $84(41.2)$ & \\
\hline Female & $40(58.8)$ & $120(58.8)$ & \\
\hline Age (years) & & & 0.60 \\
\hline $41-60$ & $20(29.4)$ & $48(23.5)$ & \\
\hline $61-80$ & $34(50.0)$ & $107(52.5)$ & \\
\hline $81-100$ & $14(20.6)$ & $49(24.0)$ & \\
\hline Socioeconomic status (thirds) & & & 0.71 \\
\hline Low & $22(34.4)$ & $55(27.1)$ & \\
\hline
\end{tabular}


Medium $18(26.5)$

$59(29.1)$

High

$28(41.2)$

$89(43.8)$

Domestic living arrangements

Cohabiting

$31(45.6)$

$105(51.5)$

Not cohabiting

$37(54.4)$

99 (48.5)

Primary disease

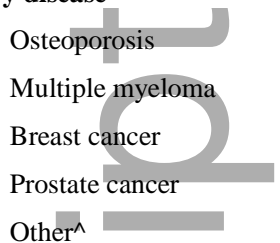

$28(41.2)$

0.97

$17(25.0)$

$8(11.8)$

$53(26.0)$

$10(14.7)$

24 (11.8)

$5(7.4)$

30 (14.7)

$11(5.4)$

Antiresorptive medication

Alendronate (Fosamax)
Zoledronic acid (Aclasta)
Zoledronic acid (Zometa)
Pamidronate (Aredia)
Risendronate (Actonel)
Denosumab (Prolia)
Denosumab (Xgeva)
Other ${ }^{\wedge}$
Combination

14 (20.6)

0.63

35 (17.2)

5 (7.4)

$16(7.8)$

$16(23.5)$

48 (23.5)

$7(10.3)$

$21(10.3)$

7 (10.3)

$21(10.3)$

4 (5.9)

13 (6.4)

$9(13.2)$

35 (17.2)

2 (3.0)

$0(0.0)$

$4(5.9)$

$15(7.4)$

Route of administration

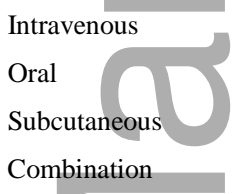

$28(41.2)$

85 (41.6)

$23(33.8)$

$56(27.5)$

$13(19.1)$

48 (23.5)

$4(5.9)$

$15(7.4)$

Nature of antiresorptive therapy

Lower dose (OP, RA or PD)

$33(48.5)$

Higher dose (oncology)

$68(51.5)$

99 (48.5)

$105(51.5)$

Duration antiresorptive therapy

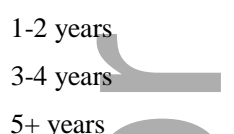

26 (38.2)

$42(20.6)$

$23(33.8)$

95 (46.6)

$5+$ years

$19(28.0)$

$67(32.8)$

Abbreviations; CML, chronic myeloid leukaemia; OP, osteoporosis; RA, rheumatoid arthritis; PD, Paget's disease

^Other included rheumatoid arthritis, Paget's disease and chronic myeloid leukaemia

$\wedge^{\wedge}$ Other included one case each of clodronate and bondronate

Table 2. Association between comorbid conditions and MRONJ disease status

\begin{tabular}{cllll}
\hline Characteristics & $\begin{array}{l}\text { Case (N=68) } \\
\mathrm{N}, \%\end{array}$ & $\begin{array}{l}\text { Control (N=204) } \\
\mathrm{N}, \%\end{array}$ & $\begin{array}{l}\text { Unadjusted } \\
\text { OR (95\% CI) }\end{array}$ & $\begin{array}{l}\text { Adjusted } \\
\text { aOR (95\% CI) }\end{array}$ \\
\hline $\begin{array}{l}\text { Antiangiogenics } \\
\text { Chemotherapy }\end{array}$ & $1(1.5)$ & $3(1.5)$ & $1.0(0.1,7.2)$ & $1.5(0.1,24.5)$ \\
No & & & & \\
Non-cancer & $31(45.6)$ & $88(43.1)$ & Reference & Reference \\
Cancer & $2(2.9)$ & $8(3.9)$ & $0.8(0.2,3.2)$ & $0.54(0.1,3.3)$
\end{tabular}

Corticosteroids 


\begin{tabular}{|c|c|c|c|c|}
\hline No & $28(41.2)$ & $96(47.1)$ & Reference & Reference \\
\hline Inhaled & $7(10.3)$ & $16(7.8)$ & $1.4(0.6,3.2)$ & $1.6(0.6,4.4)$ \\
\hline Systemic & $33(48.5)$ & $92(45.1)$ & $1.2(0.7,2.0)$ & $1.6(0.7,3.6)$ \\
\hline Diabetes & $19(27.9)$ & $26(12.7)$ & $2.0(1.2,3.3)^{* *}$ & $3.0(1.4,6.6)^{* *}$ \\
\hline Hypertension & $33(48.5)$ & $83(40.7)$ & $1.3(0.8,2.0)$ & $2.3(1.1,5.0)^{*}$ \\
\hline Dyslipidaemia & $20(29.4)$ & $50(24.5)$ & $1.2(0.7,2.0)$ & $1.1(0.6,2.3)$ \\
\hline Other cardiovascular disease & $34(50.0)$ & $60(29.4)$ & $1.9(1.2,3.1)^{* * * *}$ & $4.2(1.9,9.5)^{* * *}$ \\
\hline Gastrointestinal disease & $24(35.3)$ & $49(24.0)$ & $1.5(0.9,2.5)$ & $3.1(1.5,6.2)^{*}$ \\
\hline Respiratory disease & $27(39.7)$ & $47(23.0)$ & $1.8(1.1,2.9)^{*}$ & $1.7(0.9,3.3)$ \\
\hline Kidney disease & $15(22.1)$ & $14(6.9)$ & $2.4(1.4,4.2)^{* *}$ & $5.2(1.9,13.9) * *$ \\
\hline PTA diseases & $9(13.2)$ & $17(8.3)$ & $1.5(0.7,2.9)$ & $2.1(0.8,5.5)$ \\
\hline Blood disorders & $14(20.6)$ & $8(3.9)$ & $3.0(1.7,5.4)^{* *}$ & $5.5(1.8,16.9)^{* *}$ \\
\hline Autoimmune/Inflammatory & $17(25.0)$ & $50(24.5)$ & $1.0(0.6,1.8)$ & $1.1(0.7,1.6)$ \\
\hline Mental health & $16(23.5)$ & $33(16.2)$ & $1.4(0.8,2.5)$ & $2.1(1.0,4.4)^{*}$ \\
\hline \multicolumn{5}{|l|}{ Tobacco use } \\
\hline Not current user & $45(70.3)$ & $169(87.6)$ & Reference & Reference \\
\hline Current user & $19(29.7)$ & $24(12.4)$ & $3.1(1.5,6.8)^{* * *}$ & $3.3(1.5,7.3)^{* *}$ \\
\hline Alcohol dependency & $18(26.5)$ & 38 (18.6) & $1.5(0.9,2.6)$ & $1.1(0.5,2.4)$ \\
\hline \multicolumn{5}{|l|}{ Body mass index } \\
\hline Underweight $<18.5$ & $2(5.7)$ & $5(4.1)$ & $1.8(0.2,13.8)$ & $1.5(0.1,21.9)$ \\
\hline Normal 18.5-24.9 & $12(34.3)$ & $33(27.0)$ & $1.0(0.3,2.8)$ & $0.5(0.1,2.1)$ \\
\hline Overweight 25-29.9 & $7(20.0)$ & $44(36.1)$ & $0.3(0.1,1.1)$ & $0.1(0.0,0.7)^{*}$ \\
\hline Obese $\geq 30$ & $14(40.0)$ & $40(32.8)$ & Reference & Reference \\
\hline \multicolumn{5}{|l|}{ Systolic blood pressure } \\
\hline$\leq 130$ & $35(57.4)$ & $115(56.7)$ & Reference & Reference \\
\hline$>130$ & $26(42.6)$ & $88(43.3)$ & $1.0(0.6,1.6)$ & $1.1(0.6,2.2)$ \\
\hline \multicolumn{5}{|l|}{ Diastolic blood pressure } \\
\hline$\leq 90$ & $57(93.4)$ & $190(93.6)$ & Reference & Reference \\
\hline$>90$ & $4(6.6)$ & $13(6.4)$ & $1.0(0.4,2.8)$ & $0.6(0.1,2.3)$ \\
\hline
\end{tabular}

Abbreviations: SD, standard deviation; OR, odds ratio; PTA, parathyroid, thyroid and adrenal.

Multivariable analyses adjusted for current smoking status and duration of AR therapy.

$*$ indicates result is significant at $\mathrm{p}<0.05, * * \mathrm{p}<0.01, * * * \mathrm{p}<0.001$

Table 3. Association between CPS and risk of MRONJ

\begin{tabular}{lccccc}
\hline Characteristics & $\begin{array}{c}\text { Case }(\mathbf{N}=\mathbf{6 8}) \\
\text { Mean } \pm \text { SD }\end{array}$ & $\begin{array}{c}\text { Control }(\mathbf{N}=\mathbf{2 0 4}) \\
\text { Mean } \pm \text { SD }\end{array}$ & $\begin{array}{c}\text { Univarible } \\
\text { OR }(95 \% \mathrm{CI})\end{array}$ & $\begin{array}{c}\text { Multivariable } \\
\text { aOR }(95 \% \mathrm{CI})\end{array}$ & $\mathbf{p}$ \\
\hline All patients: & $7.2 \pm 3.0$ & $3.9 \pm 1.9$ & $1.3(1.2,1.34)$ & $2.1(1.6,2.7)$ & $<\mathbf{0 . 0 0 1}$ \\
Comorbidity Score & $13.1 \pm 4.0$ & $8.9 \pm 3.4$ & $1.2(1.1,1.3)$ & $1.5(1.3,1.7)$ & $<\mathbf{0 . 0 0 1}$ \\
Polypharmacy Score & $20.2 \pm 5.1$ & $12.9 \pm 4.6$ & $1.2(1.1,1.2)$ & $1.5(1.3,1.8)$ & $<\mathbf{0 . 0 0 1}$ \\
Combined CPS & & & & &
\end{tabular}




\begin{tabular}{|c|c|c|c|c|c|}
\hline Oncology subgroup: & $(n=36)$ & $(n=108)$ & & & \\
\hline Comorbidity Score & $5.8 \pm 2.5$ & $3.3 \pm 1.8$ & $1.3(1.2,1.5)$ & $2.3(1.5,3.6)$ & $<0.001$ \\
\hline Polypharmacy Score & $13.8 \pm 3.7$ & $9.1 \pm 3.4$ & $1.2(1.1,1.3)$ & $1.7(1.3,2.2)$ & $<0.001$ \\
\hline Combined CPS & $19.1 \pm 4.5$ & $12.4 \pm 4.5$ & $1.2(1.1,1.3)$ & $1.8(1.3,2.7)$ & 0.001 \\
\hline Non-oncology subgroup: & $(n=32)$ & $(n=96)$ & & & \\
\hline Comorbidity $\mathrm{S}$ & $8.7 \pm 2.8$ & $4.7 \pm 1.7$ & $1.4(1.2,1.5)$ & $2.0(1.4,2.8)$ & $<0.001$ \\
\hline Polypharmacy Score & $12.3 \pm 4.1$ & $8.7 \pm 3.4$ & $1.2(1.1,1.3)$ & $1.4(1.2,1.7)$ & 0.001 \\
\hline \multirow[t]{2}{*}{ Combined CPS } & $20.9 \pm 5.7$ & $13.3 \pm 4.7$ & $1.2(1.1,1.2)$ & $1.5(1.2,2.0)$ & 0.002 \\
\hline & $\begin{array}{c}\text { Case }(\mathbf{N}=68) \\
N(\%)\end{array}$ & $\begin{array}{c}\text { Control }(\mathbf{N}=\mathbf{2 0 4}) \\
\mathrm{N}(\%)\end{array}$ & $\begin{array}{l}\text { Univariable } \\
\text { OR }(95 \% \mathrm{CI})\end{array}$ & $\begin{array}{l}\text { Multivariable } \\
\text { aOR }(95 \% \mathrm{CI})\end{array}$ & $\mathbf{p}$ \\
\hline All patients: & & & & & \\
\hline Minor (0-7) & $0(0.0)$ & $31(15.4)$ & - & - & - \\
\hline Moderate (8-14) & $4(6.1)$ & $96(47.8)$ & $0.1(0.0,0.3)$ & $0.1(0.0,0.8)$ & $<0.001$ \\
\hline Severe (15-21) & $37(56.1)$ & $67(33.3)$ & Reference & & - \\
\hline Morbid (>22) & $25(37.9)$ & $7(3.5)$ & $2.26(1.36,3.76)$ & $12.7(3.2,50.0)$ & $<0.001$ \\
\hline
\end{tabular}

Abbreviations: SD, standard deviation; OR, odds ratio; CPS, Comorbidity Polypharmacy Score

Multivariable analyses adjusted for current smoking status and duration of antiresorptive therapy.

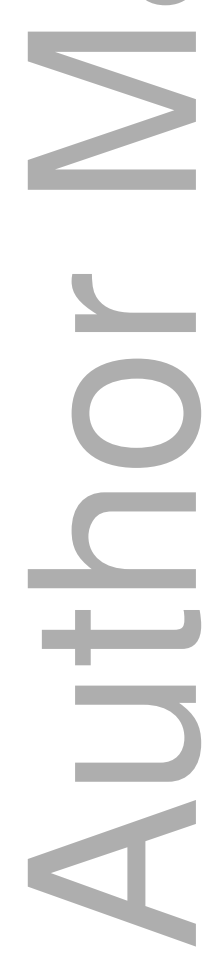

Figure 1. Distribution of cases and controls in each CPS category

[Insert Figure 1 here]

This article is protected by copyright. All rights reserved 
Figure 1 shows the unbalanced distribution of cases and controls between CPS categories.

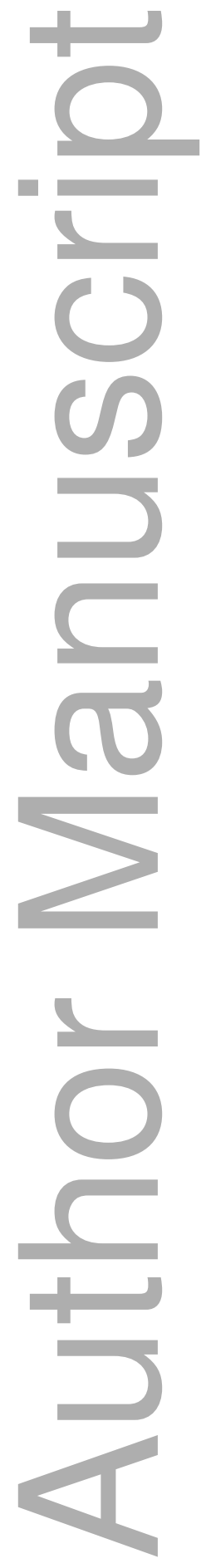




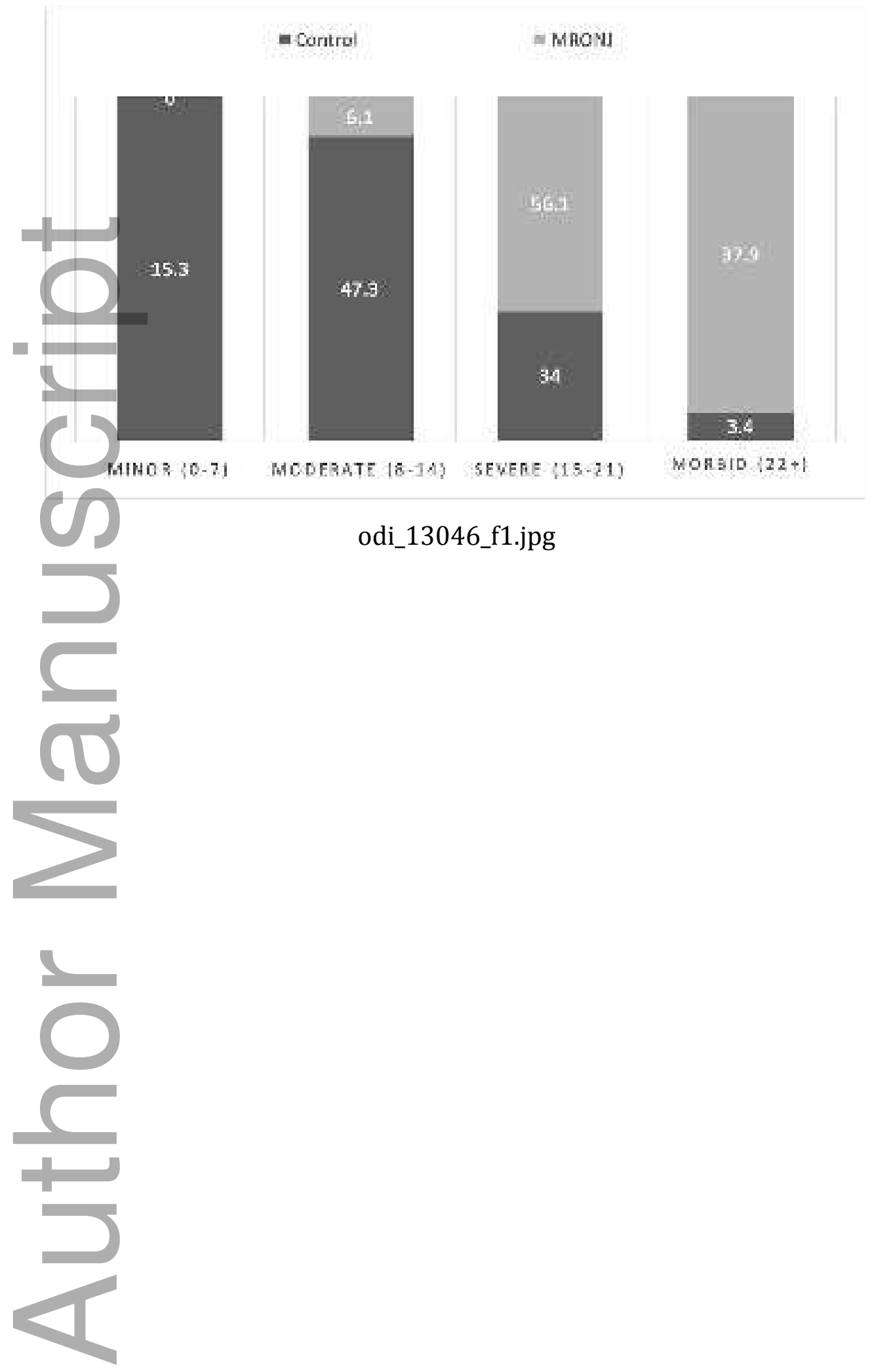

This article is protected by copyright. All rights reserved 